\title{
Considering Educators' Perceptions of Ethics and Safety in Virtual Learning Environments (VLEs)
}

Patricia B. Steele, EdD

University of Phoenix College of Doctoral Studies, Phoenix, Arizona, United States

(iD https://orcid.org/0000-0001-6533-4990

\section{Cheryl Burleigh, EdD}

University of Phoenix, Phoenix, Arizona, United States

(iD https://orcid.org/0000-0003-2393-5477

\section{Elizabeth A. Young, EdD}

University of Phoenix, Phoenix, Arizona, United States

(iD) https://orcid.org/0000-0003-3862-1323

Contact: $\underline{\text { drcburleigh@gmail.com }}$

\section{Abstract}

With the proliferation of students' abilities to use technology, in particular virtual reality (VR) applications, teachers are experimenting with VR in virtual learning environments (VLE) to engage students; but clear ethics and safety guidelines are not available. Within the field of education additional research is needed to determine how VR is employed in VLEs. Additionally, what are the decision-making processes of educators for the VR programs chosen for classroom use based on ethics and student safety? Through qualitative inquiry, educators from $\mathrm{K}-12$ and higher education shared their perceptions of ethics and safety concerns in VR applications for use in the classroom. We collected data from a sample of $\mathrm{K}-12$ and higher education professionals from the Association for Educational Communications and Technology (AECT) membership, an educational organization emphasizing technology integration and learning. Data analysis from the individual interviews with open-ended questions revealed five primary themes and twelve sub-themes. Participants agreed that they need ethical and safety guidelines as they embark on new horizons that influence student well-being. The findings from this study may provide an in-depth understanding of the importance of developing ethics and safety guidelines for educational VR programs when used by students.

Keywords: ethics; Second Life; student safety; Oculu; virtual reality; virtual learning environments

Date Submitted: September 4, 2020 | Date Published: December 1, 2020

\section{Recommended Citation}

Steele, P. B., Burleigh, C. \& Young, E. A. (2020). Considering educators' perceptions of ethics and safety in virtual learning environments (VLEs). Journal of Educational Research and Practice, 10, 404-419. https://doi.org/10.5590/JERAP.2020.10.1.26

\section{Introduction}

With the emergence of virtual reality (VR) curriculum and programs designed specifically for $\mathrm{K}-12$ and higher education, teachers may feel compelled to incorporate technology-based learning in the classroom. Teachers 
may find daunting the shift from a traditional "sage on the stage" teaching approach to developing relevant lesson plans based on increasing student engagement because they must learn how to integrate technology in the classroom quickly while learning new programs and platforms. Technology, including VR programs, has been introduced into both course curriculum and professional training applications since the 1990s. One of the earliest applications of VR for professional training was found in vertical motion simulation (VMS) training of shuttle astronauts (Beard et al., 2006). The VMS replaced traditional simulators and was used for the training of both shuttle astronauts and commercial pilots. The VMS can display various airport approaches, times of day, and weather conditions (NASA Ames Research Center, 2020). Looking forward, military and commercial pilot programs are considering the use of head-mounted VR systems for training and certification (Ellis, 2019).

In the field of education, teachers and counselors have used technology applications and programs to support student learning and social behavior. Computer-based programs found their way into schools as early as 1971 with the introduction of Oregon Trail, Where in the World is Carmen San Diego (1985), and Animated Dissection of Anatomy for Medicine (A.D.A.M.) (1990). Each of the programs was designed based on the latest technology available and presented subject content in a manner that was engaging and easy for students to understand based on age-appropriate language and concepts (Choi et al., 2016; Edutopia, 2007). When the digital classroom movement began during the Clinton Administration (West, 1995), providing computer access for all school students for educational purposes may not have been attainable. As technology has advanced, transparent integration, educational access, and applications to meet the needs of student learning and engagement have increased in both $\mathrm{K}-12$ and higher education (Baldwin, n.d.; Edutopia, 2007; Sutherland, 2020). With the proliferation of emerging technology for educational purposes and students' abilities to access computer-based gaming and social media, teachers are attempting to engage students' aptitudes in subject content-based VR applications. Two emerging VR applications in K-12 schools are Second Life and Oculus (Radianti et al., 2020) and both may pose risks to the health and safety of students based on how each is instructionally integrated.

With advances in VR technology integration for educational applications, an emphasis on end-user ethics and safety has not been considered (Checa \& Bustillo, 2019). A body of research (e.g., Adams et al., 2018; Ramirez, 2019; Sheehan \& Johnson, 2012; Weckert \& Adeney, 1997) is investigating and advocating for the accountability of ethical design and use of VR products in VLEs. Further considerations of ethics and student safety in virtual environments include the planning, assessment, and evaluation of end-user experiences (O’Connor \& Worman, 2019; Page \& Thorsteinsson, 2018). Therefore, a need exists for additional research regarding the ethics and safety of the use of VR technology for direct applications and practices within educational settings from the perspectives of educators who will incorporate VR in VLEs (Goel et al., 2016; Madary \& Metzinger, 2016). Using a qualitative inquiry approach may provide a greater understanding of how teachers are addressing issues of risk and safety and the steps needed to properly employ VR applications.

\section{Rationale and Research Questions}

Designers and developers have expressed ethical concerns regarding the proposed end-use of VR products (Adams et al., 2018; Sandler, 2016), including end-user safety, usability, and sense of presence (Radianti et al., 2020). Corporations and educational institutions implementing virtual training, for instance, have safety and liability concerns centered on the immersive experiences of the end-user (Checa \& Bustillo, 2019; Kenwright, 2018). Efforts to address usability and safety through the development of standards, procedures, and policies when integrating the use of VR for educational purposes are a concern and should be addressed prior to incorporating VR in a learning environment (Checa \& Bustillo, 2019; Goel et al., 2016; Kenwright, 2018). Carefully designed virtual training programs can enhance training and building confidence for talented students (Checa \& Bustillo, 2019; Radianti et al., 2020). As with any organization, those within educational 
settings may benefit from integrating educational applications within immersive virtual spaces where the VR programs' priorities are ethical use and safety of the products.

Although the focus of VR application and research has centered on design and end-user experiences, perceptions of educators may shed light on the ethical and safety considerations investigated by teachers prior to incorporating VR applications in the classroom curriculum. Prior to updating the curriculum, teachers may feel a responsibility to consider both the positive and negative outcomes of VR integration in classroom activities and experiences (Radianti et al., 2020; Sobel \& Jhee, 2020). The purpose of this qualitative inquiry study was to understand teachers' perceptions of the ethical and safety issues that are inherent when students are engaged in VR applications in a VLE. We interviewed seven educators who have used or who are interested in using virtual educational products in the educational environment. The following research questions were used to guide attempts to better understand the underlying phenomena within this study:

RQ1 What are educators' perceptions of their experiences in the ethical use of VR applications in educational settings?

RQ2 What are educators' perspectives of safety concerns when implementing a VR application in their classroom?

\section{Theoretical Framework}

Imenda (2014) investigated the constructs of both theoretical foundations and conceptual frameworks related to their relationship as conceptual synonyms or if they are distinct and separate constructs of synonyms. Imenda asserted that these constructs are not the same, which is evidenced and actualized within the research process. Our study, based on the construct of a conceptual framework of situated cognition, used an inductive approach and addressed the level and appropriateness of ethics in VR as total immersion educational and classroom activities.

The integration of situated cognition as the theoretical framework for this research study provided an explanation for the analysis of VR applications and ethics within educational settings. Neuman (2011) postulated that a theoretical framework, in essence, is more than a single formal or substantive theory but a combination of many theories sharing underlying assumptions and general concepts as a commonality.

The situated cognition approach, according to Koole and Veenstra (2015), places emotion regulation within the context of an activity that is based on a person's interaction with their specific environment. Heersmink (2017) focused on the moral aspects through enhancing cognitive abilities with the use of external artifacts. A prominent point of discussion pertaining to situated cognition is the relationship of the moral aspects we may term as consequences related to the brain, cognition, and culture, moral status, and a relationship to personal identity (Heersmink, 2017). Research on situated cognition must relate the concepts of phenomenological concepts of place, the embodiment of environmental factors, and immersion-in-world (Seamon, 2015).

Situated cognition provides a solid framework in the absence of research directly related to ethics and VR and provides a basis of significance to our study that is rooted in higher-order learning and development. The study may enhance the use of VR interactions within classroom learning environments when appropriate ethical realms of thought and actions are considered in developing curriculum. The study may also provide awareness to enhance the targeted professionals' cognitive abilities and connections to the moral aspects of VR classroom applications and integration.

Three areas of situated cognition, specifically embedded, grounded, and distributed cognition, are focused on the concepts of perception and action when viewed in a real-world setting through the framework of the 
human body (Dew et al., 2015). Our pursuit of further knowledge related to ethics and ethical use of VR educational applications may be perceived with a focus on observing relevant cognitive processes of an individual's mindset when developing curriculum for student use. The theoretical lens of the situated cognition framework may allow for the opportunity of new insights to be gained based on the integration of new technologies related to VR in virtual learning environments (VLE) and its applications.

The situated cognition conceptual framework presents a supporting theory for the potential ethical issues as related to VLEs. Choi et al. (2016) addressed the use of human subjects, the use of both private and public internet spaces and communities, concern and issues related to standards, guidelines, and policies of immersive technologies; and most significantly, ethical considerations for future educational and $\mathrm{K}-12$ research. The findings of Choi's study, therefore, could directly apply to the areas of correlation and significant need within VLEs. The research community is faced with increasing and varied concerns related to technology use standards and this study, through the situated cognition theoretical framework, will investigate, explore, and draw conclusions for appropriate ethical standards for VLEs.

Schott and Marshall (2018) described the theoretical framework of the situated experimental environment focusing on the impact of virtual environments on users' experiences. Further, they posited that the experiences' pedagogical value becomes enabled by the immersion process of interactions with others and a reality-based environment. While immersive learning environments are easily attained, achieving high stages related to interactivity is a challenge. The pedagogical value of such experiences is enabled through immersion in a reality-based environment, engagement with complex and ambiguous situations and information, and interaction with the space, other students, and teachers (Schott \& Marshall, 2018; van Ginkela et al., 2019). The results demonstrate that complex immersive learning environments are readily achievable but that high levels of interactivity remain a challenge. Maintaining acceptable levels of moral aspects and ethical behavior is an area to further investigate as the challenge of levels of interactivity continues to be explored.

Hashim et al. (2018) examined the development of a conceptual framework that demonstrated numerous layers, which include the format of the presentation, the informational content, the functionality of the service, the actual interactivity, augmentation of visual material, and the mobility of the technology in use. Ethics becomes a focused area of consideration for the development of a curricular framework based on the variance that may be encountered in different types of presentation and the levels of interactivity (van Ginkela et al., 2019). Changes in the components of informational content and the complexities of such may also need to be addressed when considering the intended audience. A level of acceptable ethical aspects should be determined and standards set based not only on the end-user but also the intended purpose of the augmented reality or VR product in a VLE.

From a historical perspective, Slater and Wilbur (1997) addressed how the concepts of both immersion and presence in virtual settings and environments may be assessed in an objective manner through the characteristics of the technology in use. The related aspects of inclusivity, extensiveness, surrounding, and how the virtual environment is displayed to a participant are additional considerations when developing curriculum for an immersive experience. The state of presence can be defined in this type of setting as to how it is associated with various immersion dimensions serving as a baseline of moral pillars for VR and VLEs.

\section{Recruitment, Methodology, and Data Analysis}

We used a qualitative inquiry approach to elicit responses to the two research questions that we derived from the problem under study and the purpose. Participants were recruited from the Association for Educational Computers and Technology (AECT), a professional international educational organization, by advertising to the group the research team's request for participants in the study to learn about their perspectives on the 
ethical use of VR applications and to discuss how they protect the health and safety of their students when using the VR applications.

For consistency and to maintain trustworthiness and accuracy in the format used to interview study participants, the team agreed to an interview protocol that would standardize the interview process. It was also agreed that one researcher would conduct telephone interviews and record and transcribe them. Participants checked their transcripts for accuracy and returned them to the interviewer. Content analysis was done independently by all research team members. The content analysis was followed by a consensus of opinions on the main themes that emerged from the participants' interview transcripts (Krippendorff, 2018). Reporting of the findings began with an explanation of the two main applications that are used in the classroom: Second Life and Oculus.

\section{Instrumentation}

As in all qualitative research involving one-on-one interviews, the main instrument is the researcher asking the questions. The research team put together an interview protocol that would be used for each interview in order to standardize and meet the requirements of accuracy and credibility; thereby, strengthening the trustworthiness and replicability of the study (Garbarski et al., 2016). Confidentiality forms were signed by each volunteer study participant giving permission to be audio recorded by the interviewer. Each participant was given a pseudonym. The pseudonym was the only form of identification used throughout the study.

\section{Population and Sample}

The population for this qualitative inquiry study were the approximately 2000+ members of AECT. Initially, a description of the study was announced through the AECT website. We sought participants for this study who were educational specialists, trainers, or teachers in $\mathrm{K}-12$ or higher education and had either worked or taught in the field of education for a minimum of three years. They had to have used, planned to use, or had experience with VR in a classroom with students. Those expressing interest then were sent an invitation letter giving details of the study and an Informed Consent letter to be signed and returned to the researcher, if interested in participating in the one-on-one phone interviews. From the initial 20 potential study participants who responded to the research solicitation, seven committed to being interviewed. Those individuals who did not commit to the interview may not have qualified based on the outlined participant criteria or, if they did meet the study's requirements, self-selected not to proceed to the interview phase of the study.

The population of study participants consisted of three females and four males, five of whom were teachers in higher education, one a $\mathrm{K}-5$ educator, and one individual who was a $\mathrm{K}-12$ education trainer. Each of the study participants had experience in VR applications for use in VLEs. This criterion allowed the researchers to capture the participants' insights and experiences regarding the ethical considerations of employing VR in curriculum development and use in VLEs.

\section{Data Collection and Analysis}

The lead researcher on this team conducted all of the interviews over the telephone to ensure consistency and standardization of the interview protocol. Open-ended questions were used to gain perceptions of each study participant with a focus on ethics of care in the use of VR applications within VLEs. The questions sought to understand participants' role in deciding which program to use and the suitability of the VR application for the student population.

Follow up questions allowed the researcher to ask additional questions and delve deeper into the responses provided by the participants as to ethics or other situations in which ethical considerations may be considered in the use of VR in a VLE. One researcher served as the instrument for the study to collect the participants' 
stories through one-on-one interviews. Participant interviews were digitally recorded and transcribed by a researcher. The same researcher transcribed the audio recordings and sent the Word documents back to each of the participants for member checking (Clandinin \& Connelly, 2000; Kim, 2016). Content analysis took place using a modification of Creswell and Creswell's (2018) four steps of qualitative data analysis process. The steps used for the analysis of the data included the organization of data and creation of files; reading and note-taking of transcripts for common thought patterns and themes; describing the data using common phrases and words; and classifying the data into themes to identify key parts of the participants' stories from the transcripts, locating researcher-identified key terms and thoughts, and then supporting that data with contextual notes from the transcripts (Creswell \& Creswell, 2018). The individual team members analyzed each transcript and then collectively discussed them several times. As to the participant's story, we identified emerging codes, noting categories, followed by specific themes and sub-themes. The specific themes identified included psychological safety, social safety, physical safety, ethical morality, and teacher responsibility. Subthemes were identified in each of the specific themes and included emotional safety, disengagement, protection of personal identity, respect for other avatars, awareness of hackers, Oculus, instructor, diversity, expectations, monitoring, parent permission, and safety and well-being.

\section{Results and Discussion}

The use of VR in VLEs led to a discussion of specific program applications for educational purposes. The two VR programs, Second Life and Oculus, were discussed in the context of this study with each of the participants. Second Life and Oculus were actively being used by three of the seven participants. All but one participant, P1, had either used or were investigating using Second Life. Three of the participants were actively engaged in using Second Life and had discussed specific details about the program, which we thought were worth mentioning prior to the themes.

Participant $\mathrm{P}_{3}$ found that since she had to incorporate Native American Indian content in her basic media course, using Second Life could supplement the required content.

My course doesn't have any content but how to turn the equipment on. I realized I also wanted to get them [the students] into the virtual world to see how that could be used. I looked into Second Life to see if I could find Native American content there, which I did find. We had media students go in and look at that.

When asked about the ethics of using Second Life in the media class, P3 replied,

It would be the same as ethics in education anywhere else. To teach instructional media, one of the points I tried to make to people is whenever some new tool comes along, it looks like it's weird, strange, and it's going to change everything, but in fact, it just turns out to be a tool for teaching. We have a professional code of ethics in [my state]. The professional code of ethics and rules applies for the virtual world as well. Safety concerns come to mind because in the virtual world, it's like taking students on a field trip in a place where absolutely anything might show up. And so you have to do certain things to make sure the students are safe in those circumstances. There's something, anytime you choose a particular medium to use, you have to be aware of the fact that it's going to work better for some students than for others. And my experience in taking people into a 3 Dimensional world has been that some people take to it immediately and others understand what's going on here and what could be done here. Other people who are indifferent to it, and some people have a very negative reaction to it. They see it as an attempt to withdraw them from reality, or to expose them to something dangerous, and you have to be ready to work with those folks, to either help them to understand what's going on there, or to have some alternative that they can be doing instead of that situation that they see as hostile to them. 
$\mathrm{P}_{3}$ discussed the topic of safety issues as a concern that involved using any VR program. With regards to Second Life, depending on the ages of the students and level of maturity, students need to be informed prior to the use of the program. $\mathrm{P}_{3}$ stated as the instructor there is an advantage that you have complete control over what is shown in the program and how the scenarios may possibly play out. With every advantage, $\mathrm{P}_{3}$ indicated there are disadvantages.

You do not get to show the students the wonderful things that are out there in the wide virtual world for them to see. If you want to take them out into that world, they have to be prepared for it. They have to know the fact that it's possible that they will encounter someone out there who may not have their best interest at heart. Which is true if you take students out into the real world on a field trip. In the virtual world it's easier because in the virtual world you have so much more opportunity to have contact with people at tremendous distances with complete anonymity on their part.

A participant who also used Second Life, $\mathrm{P}_{7}$, was a trainer for pre-service Chinese and American teachers. $\mathrm{P}_{7}$ shared that during training, "all the activities were oriented around language and cultural learning about Chinese and American cultures.” P7 worked with graduate students.

Who served as mentors or instructors in the virtual world of Second Life? They taught kids, and professors from China and also me, a professor from the United States, how to monitor activities in Second Life. We recorded their instruction and then watched the recording together with the students. The reflection and comments were part of learning how to properly interact and deliver instructions in virtual reality across the different cultures. $\sim \mathrm{P} 7$

P4 worked with students in higher education who were pursuing social work. P4 would use Second Life as a facilitation tool for students to learn how to conduct group sessions and interaction with diverse populations they may encounter as a social worker. "The students were into the virtual reality of Second Life. They took on the role as social workers to facilitate group discussions, which led to doing activities together in the virtual reality space."

Finally, when asked about ethical decisions required when creating VR scenarios, P4 responded, "it is the decisions you make when you want no harm, you are equitable, fair, looking at the student and making sure they are safe, psychologically, physically, morally, they are ethically safe in those experiences with VR.”

One participant, P6, reported that they used a modification of Second Life in her school. The program was placed behind the school server's firewall. For her classes, P6 needed to recreate Second Life with a simulator using open Avatars. Parents were required to sign consent forms for each child using the program. P6 said that the program was built carefully to avoid any ethical issues. The students were asked about their prior experiences with Second Life or other VR programs. Students were also involved in the designing of the projects within the simulator. Students were directed by P6 on how to safely use the VR program, including what to do if they felt dizzy from the movement, how to stop the VR experience or to report a situation that felt uncomfortable at any time. After the sessions, P6 would hold a debriefing session with each class.

The following categories emerged from the content analysis of the participant's transcripts. The themes were centered on the five categories of psychological safety, social safety, physical safety, ethical morality, and teacher responsibility. The excerpts below are from the seven participants who are representative of the specific data category.

\section{Psychological Safety}

The following excerpts from the data show that teachers have ongoing experiences and concerns with the possible effects of VR on students' psychological safety. Psychological safety included the sub-themes of 
emotional safety and disengagement. Of note, teachers discussed psychological and emotional safety in concert with teacher responsibility. Disengagement was seen by teachers as a proactive measure to disconnect from the VR activity to protect students' psychological and emotional well-being.

P2 shared that it was the instructor's responsibility to be aware there "could be some kind of emotional experience with students that we cannot predict. One can never predict the effect of a VR experience on an individual either psychologically or emotionally."

My experience in taking people into a three-dimensional world has been that some people take to it immediately and other people are indifferent to it. Either set of students may have a very negative reaction to the VR experience. If emotional safety or psychological safety is a concern, then the VR experience needs to be terminated. $\sim \mathrm{P} 3$

I embarked on building my own VR system, which would be protected by the school firewall to help reduce any potential emotional issues with students. $\sim \mathrm{P} 6$

P1 explained that she talks to the students about what to do when something pops up within the VR experience that scares them or makes them feel uncomfortable. "When something pops up on the screen that you don't want to see or you know that you shouldn't be seeing, disengage from the internet immediately and let me know what you have seen." P1 reinforces with her students "to call over a friend, and then have a conversation about it with me." She added, "that's when the student speaks with a friend about the experience, they discuss that they went to this place in the VR experience and what comes up, which makes them uncomfortable, then asks the question, what should I do?”

P3 instructed students on how to escape an uncomfortable or threatening situation while online in the VR activity by using the "h" key on their computers. He explained to them, "You have a key command that you can transport yourself home."

These responses illustrate the types of lived experiences of educators and students when using VR in a VLE activity. Information on the possible emotional or psychological effects of VR is not a point of discussion when programs are advertised to enhance student learning and are an ethical concern of teachers. Only through inclass experiences are teachers' awareness heightened on the possible effects of VR activities in the classroom.

\section{Social Safety}

When discussing social safety, four of the study participants made connections between the protection of personal identity, respect of avatars, and awareness of hackers, indicating these phenomena are not just isolated within a single VLE. The following excerpts are indicative of those conversations.

My biggest concern for safety for the kids is that it is not appropriate to give their name, address, phone number, or meet up in person with someone they meet online. Students need to learn how to protect their identity and this is one thing that we need to teach them, especially when they are online. $\sim \mathrm{P} 1$

Instructors need to be cognizant when working with certain VR software platforms [such as] OCULUS. As instructors, we need to be aware of whether any data is being collected, generated, and shared back to the corporations and be open about that as well with the school, parents, and students. It is possible for personal data to be captured in that environment experience, so we have to always be aware of that. It is part of our ethical use to share that. $\sim \mathrm{P} 2$

Some students see using VR as an attempt to withdraw from reality, or to expose themselves to something dangerous. You have to be ready to work with those folks, to either help them to 
understand what's going on there, or to have some alternative activity that they can be doing instead of that situation that they see as hostile or dangerous to them. $\sim \mathrm{P} 3$

I want to make sure that my students understand that even though it is virtual reality and they are acting through their avatars, there are the real people behind those avatars. So being respectful is one thing that I require for my students. If I observe wrong or inappropriate behavior, I stop it immediately. $\sim \mathrm{P} 4$

I am concerned about my students' identity being compromised in the VR environment when they are on the web. Students have to know that it's possible they will encounter someone out there who may not have their best interest at heart. Which is true if you take students on a real-world field trip. In the virtual world it's easier [for hackers to compromise students' identity] because in the virtual world you have so much more opportunity to have contact with people at tremendous distances with complete anonymity on their part. $\sim \mathrm{P}_{3}$

I warn my students that even though there are real people behind the avatars and they, as students, must be respectful, there is always the possibility there could also be hackers who break through the firewall and pretend to be someone they are not. $\sim \mathrm{P} 4$

Teachers take the responsibility of student social safety seriously, especially when introducing students to curriculum and activities that are based in VR. Although teachers at all levels hope the learning environment will be free from hazards that may affect students, each teacher expressed the need to be guarded and to continuously consider the students' well-being in any VR activity undertaken in a VLE.

\section{Physical Safety}

In the participants' discussions, one of the challenges expressed was in the use of Oculus headsets. Each of the participants' experiences in relation to student use of Oculus differed, yet the need to be diligent in the use, length of time, and exposure to the headsets was a concern.

A concern if there is a student who doesn't want to participate in VR, like putting on the headset, I am thinking about how to navigate that. Not wanting to force them to do that but if there is strong value in participating in that activity, how do you encourage participation without making anybody feel uncomfortable? $\sim \mathrm{P} 2$

OCULUS can pose safety issues. The system that I have is not attached to anything. The manufacturers suggest making a VR zone and coning it off. Making it very clear and making sure you're there with the user so they don't leave the zone or hurt themselves. $\sim \mathrm{P} 2$

Going into a world with a VR headset, you really feel that you are there. For some people it's going to help to get through that boundary so that they feel they have gone to another place. But for other people who are really upset by the VR experience, though, that's just going to make it worse for them. $\sim \mathrm{P}_{3}$

A concern is the length of time that the kids are wearing those headsets. I don't know. I just feel sometimes it might cause damage to their vision. Wearing too long, that could be harmful. $\sim \mathrm{P} 4$

Everything in the VR environment must be controlled for safety at all times. There can be disorienting experiences. It can appear that the floor opens or moves causing someone to fall. $\sim \mathrm{P}_{3}$

If I'm going to use a VR product, I would conduct research on the safety of the product-physical safety and psychological safety, what is it made of so that if students have to wear the product for 
maybe thirty minutes, it doesn't cause any harm to their face, or wherever they have to wear it on their body, and the product will not collect information or present information that has as not been divulged previously of the participants. $\sim \mathrm{P} 7$

\section{Ethical Morality}

Participants expressed concerns regarding ethics and moral philosophy with the topic embedded in other themes presented and in the discussion of Second Life. The following statements capture the essence of the discussion of ethics and diversity in the use of VR in VLEs independent of what has already been presented.

Ethics in a virtual space really boils down to your philosophy in education. I mean it really has to do with what is your endgame or the goal for your kids. There can be ethical situations, but it really depends on how you present them and how those are handled when they occur. $\sim \mathrm{P} 1$

In terms of ethics, we have to think of any sort of student information or confidentiality and consider that before we would capture those images when using the OCULUS system. $\sim \mathrm{P} 2$

It is about the decisions you make that are equitable, fair, and making sure they are safe, psychologically, physically, morally, they are ethically safe in those experiences with VR. $\sim \mathrm{P} 4$

Ethical concerns, a number of things that come up from the application perspective. Some things that I think would be interesting to do with it [VR] would be around diversity, giving people alternative perspectives for a better sense of empathy. I was talking with a colleague about having to walk through a day as a race or gender different from your own. Or to create a VR experience to provide students with opportunities to experience what it was like to be blind, deaf, or handicapped physically. $\sim \mathrm{P}_{5}$

Ethical concerns in VR for educational settings are really based on the design and the designer environment. To use VR we have human ethics as a committee permission and we make sure when we inform whoever participates in those sessions what may be the complications prior to starting. We also tell them that they disengage at any time. $\sim \mathrm{P} 6$

I feel strongly about a user's or participants' rights. What they need to know to participate and be $100 \%$ informed with no surprises. The reason I feel that way, especially when working with kids. In the past, I saw teachers collecting information without permission about the students' behavior, they and their parents were not aware. Using information or engaging in activities, especially when using VR, without permission, doesn't seem right to me. Everything should be open; nothing should be hidden. $\sim \mathrm{P} 7$

\section{Teacher Responsibility}

There was agreement among participants that teachers are responsible for how to correctly employ VR in the classroom as to student expectations and correctly monitoring student use. In particular, safety and the wellbeing of students were imperative to successful VR experiences in a VLE. Participants agreed before embarking on any VR teaching strategy, the students must be made aware of what was going to happen and given the opportunity to opt-out of the venture and accept an alternate assignment. Participants were cognizant of making sure that the VR experiences were age-appropriate. P4 cautioned, "we have to really closely monitor where they [the students] are in virtual worlds and what they do there."

Continuous monitoring [of students] is challenging, and apps are used, such as class DOJO, which is a very safe place. People cannot even get on it and access that unless they have been given a code by their teacher. $\sim \mathrm{P} 4$ 
When monitoring student use, all of a sudden, you may realize that some of the kids are not with you anymore. When you look, they are a place they are not supposed to be. And you have to drag them off from that location. I always have to remind them about the places they are not to go during the class." $\sim \mathrm{P} 4$

For participants who work with younger students, parental permission is needed to engage in VR-based activities. One participant, P1, shared, "we do have a safety policy that the parents complete at the beginning of the school year. If parents haven't filled out the paperwork, students won't have access to devices, or they would have limited use of technology."

I am always concerned about safety. I am working with a vulnerable population. If I am going to present a lesson, I am either going to be making the activities and material myself or preview the material before I give approval. $\sim \mathrm{P} 1$

In some instances, safety measures and school policy were not readily available nor readily visible to parents and teachers. One such instance was described by P1.

I went to the website and found a Code of Conduct and Policies and couldn't find anything. Then I spoke to others in my department, and I'm being directed to like the technology office on campus, like Vice Provost and Ed Technology Services. I can tell you; it does not appear readily available.

When monitoring student use, $\mathrm{P}_{5}$ described VR as a "sandbox world where people just play with an alternate reality. I think one of the main objectives of VR instructional strategies would be to help build critical thinking and ethical behavior."

P6 embarked on building her own VR system, which would be protected by the school's firewall. The VR system, OpenSim, allows instructors to have complete control and build the entire VR experience.

If students have any unpleasant sensation, they can leave Sim anytime they want. They can try it and create their own avatar if they are curious, but if not, then they just don't. Even though we [as teachers] have created what we feel is the safest VR Sim experience for their students, I still express doubts that anything could be $100 \%$ safe, and teachers need to be alert and aware of all caveats. $\sim$ P6

\section{Summative Statement}

While the participants in this study were either providing VR experiences for their students or researching various VR programs, they were all genuinely cautious about the ethical approach they would take to implement VR in a VLE. Their main concerns were the safety of the students in the areas of emotional, physical, and social safety. One participant summed up the concern that participants had when using VR teaching strategies, and asked, "where are the guidelines for novice users and experienced users to follow?" This research team is quick to respond to the need for guidelines and has begun to pull together their thoughts and notes to write guidelines for teachers using VR in the classroom based on the experiences of teachers in the field who have journeyed into a possible black hole of unknown expectations.

\section{Significance}

Contributions of this study could bring awareness of ethical considerations to educators, administrators, and curriculum specialists in the fields of pedagogical planning and instruction. Thoughtful consideration of this study's results in the use of VR in VLEs could encourage those who have direct influence to adopt VR programs to investigate various ethical aspects of the application on the end-user, the student. A potential exists for safer, more thoughtful planning and oversight when implementing VR experiences in educational 
settings. Planning, procedures, and decisions regarding ethics in VLEs may affect individuals within the organization physically, psychologically, and socially. We hope that this study will be used to increase awareness of the need for ethical considerations when planning, applying, assessing, and evaluating VR educational applications in VLEs. A deeper understanding of VR ethical considerations based on student use and well-being is encouraged through expanded research.

\section{Limitations}

There were several limitations to be considered for this study. The first limitation of this study pertains to the generalizability of the sample, which included a relatively small number of participants, a total of seven, from a population of approximately 2,000 members from a single professional international educational organization, AECT. Participation in this study was voluntary and self-selected. The sample is adequate for the purposes of this research methodology (Boddy, 2016); the inclusion of participants that were not members of this specific professional organization were not considered. Although the number of participants was smaller than expected, the research team agreed that saturation was reached through data obtained during each of the participants' interviews. Even though participants invited to take part in the study were from a single educational organization, future studies could include participants from multiple professional organizations, practitioners in the field, which represent various types of schools in $\mathrm{K}-12$ and higher education. In addition, further studies could use snowball sampling as a method to solicit and select participants. A second limitation, since the data was collected from a convenience sample from a single professional international education organization, we do not know whether our findings would be generalizable to other educators who are using or will consider implementing VR in VLEs in the United States or internationally.

\section{Recommendations}

Recommendations for future research may include ethical studies concentrating specifically on the four themes identified in this study. Each individual study may reveal in-depth information for specific issues, problems, and possible solutions regarding virtual experiences in an immersive VLE. Further research could be conducted regarding physical, psychological, moral, and social issues that may arise in the use of VR with students in $\mathrm{K}-12$ or higher education and how concerns for student safety in these areas can be addressed. Individuals who may participate in such studies could include teachers who have prior experience in the use of VR products and curriculum that is specifically designed for educational settings inclusive of studentcentered learning and VLEs. These same teachers may previously have been encouraged to proactively plan, apply, reflect, access, and evaluate the virtual experiences to be offered in the classroom (Johnston et al., 2017; Patterson \& Han, 2019). Experienced education practitioners may provide greater insight to existing data on ethical practices of VR and how such programs are used with diverse student populations.

Additionally, educators in $\mathrm{K}-12$ or higher education may be able to discuss observations based on prolonged student interaction with VR educational programs and how such experiences affected students' psychological and physical safety and what social or moral issues may have arisen from increased usage of those programs in a VLE. It is recommended that a specific, in-depth curricula guide be developed to assist educators who are faced with planning VR experiences for their students. This guidebook may include information for planning, applying, reflecting, assessing, and evaluating when implementing VR in a VLE setting (Johnston et al., 2017; Patterson \& Han, 2019). These guidelines should be available in $\mathrm{K}-12$ and higher education for engaging educational training of faculty throughout the educational institution. 


\section{References}

Adams, D., Bah, A., Barwulor, C., Musabay, N., Pitkin, K., \& Redmiles, E. (2018, August). Ethics emerging: The story of privacy and security perceptions in virtual reality. Fourteenth Symposium on Usable Privacy and Policy. August 12-14, 2018. Baltimore, MD. https://www.usenix.org/system/files/conference/soups2018/soups2018-adams.pdf

Baldwin, R. G. (n.d.). Technology in education: Higher education. https://education.stateuniversity.com/pages/2496/Technology-in-Education-HIGHEREDUCATION.html

Beard, S. D., Ringo, L. A., Mader, B., Buchman, E. H., \& Tanita, T. (2006). Space shuttle landing and rollout training in vertical motion simulator. AIAA Modeling and Simulation Technology Conference. https://doi.org/10.2514/6.2008-6541

Boddy, C. (2016). Sample size for qualitative research. Qualitative Market Research, 19(4), 426-432. https://doi.org/10.1108/QMR-06-2016-0053

Checa, D., \& Bustillo, A. (2019). A review of immersive virtual reality serious games to enhance learning and training. Multimed Tools and Applications, 79, 5501-5527. https://doi.org/10.1007/s11042-019$\underline{08348-9}$

Choi, D. H., Dailey-Hebert, A., \& Estes, J. S. (Eds.). (2016). Emerging tools and applications of virtual reality in education. Information Science Reference.

Clandinin, D. J., \& Connelly, F. M. (2000). Narrative inquiry: Experience and story in qualitative research. Jossey-Bass.

Creswell, J. W., \& Creswell, J. D. (2018). Research design: Qualitative, quantitative, and mixed methods approaches (5th ed.). SAGE.

Dew, N., Grichnik, D., Mayer, H. K., Read, S., \& Brinckmann, J. (2015). Situated entrepreneurial cognition. International Journal of Management Reviews, 17(2), 143-164. https://doi.org/10.1111/ijmr.12051

Edutopia. (2007, November 5). What is successful technology integration? https://www.edutopia.org/technology-integration-guide-description

Ellis, C. (2019, September 10). Are VR flight simulators the future of pilot training? Air Charter Service. https://www.aircharterservice.com/about-us/news-features/blog/are-vr-flight-simulators-thefuture-of-pilot-training

Garbarski, D., Schaeffer, N. C., \& Dykema, J. (2016). Interviewing practices, conversational practices, and rapport: Responsiveness and engagement in the standardized survey interview. Sociological Methodology, 46(1), 1-38. https://doi.org/10.1177/0081175016637890

Goel, A., Rivera, W. A., Kincaid, P., Montgomery, M., Karwowski, W., \& Finkelstein, N. M. (2016). Ethics in virtual world environments research. In H. W. Choi, A. Dailey-Hebert, \& Simmons Estes (Eds.), Emerging Tools and Applications of Virtual Reality in Education (pp. 258-276). IGI Global.

Hashim, M. E. A. H. B., Idris, M. Z. B., \& Othman, A. N. B. (2018). Developing theoretical framework on augmented reality (AR) comic using user experience (UX) approach. International Journal of Academic Research in Business and Social Sciences, 8(2), 844-851.

Heersmink, R. (2017). Extended mind and cognitive enhancement: Moral aspects of cognitive artifacts. Phenomenology and the Cognitive Sciences, 16(1), 17-32. https://doi.org/10.1007/s11097-015-9448-5

Imenda, S. (2014). Is there a conceptual difference between theoretical and conceptual frameworks? Journal of Social Sciences, 38(2), 185-195. https://doi.org/10.1080/09718923.2014.11893249 
Johnston, E., Oliva, G., Steele, P., Smith, C., \& Bailey, L. (2017). Exploring pedagogical foundations of existing virtual reality applications: A content analysis study. Journal of Educational Technology Systems, 46(4), 414-139. https://doi.org/10.1177/0047239517745560

Kenwright, B. (2018). Virtual reality: Ethical challenges and dangers. IEEE Technology and Society Magazine, 37(4), 20-25. https://ieeexplore.ieee.org/document/8558774

Kim, J. H. (2016). Understanding narrative inquiry. SAGE.

Koole, S. L., \& Veenstra, L. (2015). Does emotion regulation occur only inside people's heads? Toward a situated cognition analysis of emotion-regulatory dynamics. Psychological Inquiry, 26(1), 61-68. https://doi.org/10.1080/1047840X.2015.964657

Krippendorff, K. (2018). Content analysis: An introduction to its methodology. SAGE Publications.

Madary, M., \& Metzinger, T. K. (2016). Real virtuality: A code of ethical conduct. Recommendations for good scientific practice and the consumers of VR-technology. Frontiers in robotics and AI, 3(3), 1-23. https://doi.org/10.3389/frobt.2016.00003

NASA Ames Research Center. What is the vertical motion simulator? https://www.nasa.gov/ames/vms

Neuman, W. L. (2011). Social research methods: Qualitative and quantitative approaches (7th ed.). Allyn \& Bacon.

O’Connor, E. A., \& Worman, T. (2019). Designing for interactivity, while scaffolding student entry, within immersive virtual reality environments. Journal of Educational Technology Systems, 47(3), 292-317. https://doi.org/10.1177/0047239518817545

Page, T., \& Thorsteinsson, G. (2018). Designing to support children development: A literature review. I-manager's Journal on Educational Psychology, 12(1), 32-42. https://doi.org/10.26634/jpsy.12.1.14605

Patterson, T., \& Han, I. (2019). Learning to teach with virtual reality: Lessons from one elementary teacher. Tech Trends, 63, 463-469. http:/doi.org/10.1007/s11528-019-00401-6

Radianti, J., Majchrzak, T. A., Fromm, J., \& Isabell Wohlgenannt, I. (2020). A systematic review of immersive virtual reality applications for higher education: Design elements, lessons learned, and research agenda. Computers \& Education, 147(103778). https://doi.org/10.1016/j.compedu.2019.103778

Ramirez, E. J. (2019). Ecological and ethical issues in virtual reality research: A call for increased scrutiny, Philosophical Psychology, (32)2, 211-233. https://doi.org/10.1080/09515089.2018.1532073

Sandler, R. (Ed.). (2016). Ethics and emerging technologies. Springer.

Schott, C., \& Marshall, S. (2018). Virtual reality and situated experiential education: A conceptualization and exploratory trial. Journal of Computer Assisted Learning, 34(6), 843-852. https://doi.org/10.1111/jcal.12293

Seamon, D. (2015). Situated cognition and the phenomenology of place: Lifeworld, environmental embodiment, and immersion-in-world. Cognitive processing, 16(1), 389-392. https://doi.org/10.1007/s10339-015-0678-9

Sheehan, M., \& Johnson, R. (2012). Philosophical and methodological beliefs of instructional design faculty and professionals. Educational Technology Research and Development, 6o(1), 131-157. www.jstor.org/stable/41415021

Slater, M., \& Wilbur, S. (1997). A framework for immersive virtual environments (FIVE): Speculations on the role of presence in virtual environments. Presence: Virtual and Augmented Reality. https://doi.org/10.1162/pres.1997.6.6.603 
Sobel, K., \& Jhee, C. (2020). How K-8 teachers are using virtual and augmented reality in classrooms today. CIRCL Project Spotlight Series. https://circlcenter.org/how-k-8-teachers-are-using-vr-and-ar-inclassrooms-today/

Sutherland, C. (2020, March 27). 7 reasons why students need technology in the classroom. Explorance. https://explorance.com/blog/7-reasons-students-need-technology-classroom/

van Ginkela, S., Gulikersb, J. T., Biemansb, H. J. A., Noroozi, O., Roozenc, M., Bosd, T., van Tilborge, R., van Halterene, M., \& Mulder, M. (2019). Fostering oral presentation competence through a virtual reality base task for delivering feedback, Computers and Education. 134, 78-97.

https://doi.org/10.1016/j.compedu.2019.02.006

Weckert, J., \& Adeney, D. (1997). Computer and information ethics. Greenwood Publishing Group Inc.

West, P. (1995, October 18). Clinton pushes schools-technology campaign. Education Week. https://www.edweek.org/ew/articles/1995/10/18/o7tech.h15.html about developments and change in the field of education and learning. The journal includes research and related content that examine current relevant educational issues and processes. The aim is to provide readers with knowledge and with strategies to use that knowledge in educational or learning environments. JERAP focuses on education at all levels and in any setting, and includes peer-reviewed research reports, commentaries, book reviews, interviews of prominent individuals, and reports about educational practice. The journal is sponsored by the Richard W. Riley College of Education and Leadership at Walden University, and publication in JERAP is always free to authors and readers. 